\title{
Impacto socioeconómico y la gestión de los residuos sólidos en el distrito de Moche, Trujillo - 2021
}

\author{
Socioeconomic impactand solid waste management in the district of \\ Moche, Trujillo - 2021
}

Recibido: julio 25 de 2021 | Revisado: setiembre 20 de 2021 | Aceptado: octubre 19 de 2021

\section{Edit Marleni Pérez Morales ${ }^{\mathrm{I}}$}

1 Escuela de Posgrado, Universidad César Vallejo, Trujillo

Autor para correspondencia

E-mail: marleniperez@gmail.com

\begin{abstract}
Resumen
En la presente investigación se tuvo como objetivo general determinar las características del Impacto Socioeconómico de la gestión de los residuos sólidos en el distrito de Moche, Trujillo - 2021.Se realizó la respectiva inspección metódica de la literatura científica de los últimos cinco años respecto al Impacto Socioeconómico de la gestión de los residuos sólidos. Se halló que en la localidad de Pereira el 26\% de los encuestados no tiene conocimiento de lo que sucede con los residuos sólidos y que solo un 3\% supone que dichos residuos terminan siendo reciclados, mientras que el municipio de Haina el $48 \%$ reconoce no tener información respecto al impacto de las situaciones ambientales y socioeconómicas. Se tuvo como principal conclusión que el desconocimiento de un inadecuado manejo de los residuos sólidos puede traer consecuencias lamentables en el ámbito económico, social y administrativo. Por lo que es necesario el apoyo por parte de las autoridades y población en general.
\end{abstract}

Palabras clave:impacto socioeconómico; gestión de los residuos; factor demográfico; factor social y educación ambiental

\begin{abstract}
The general objective of this research was to determine the characteristics of the Socioeconomic Impact of solid waste management in the district of Moche, Trujillo - 2021. After conducting the respective methodical inspection of the scientific literature of the last five years regarding the Socioeconomic Impact of Solid waste management, it was found that in the town of Pereira, $26 \%$ of those surveyed have no knowledge of what happens with the solid waste and that only $3 \%$ suppose that said waste ends up being recycled, while the municipality of Huaina $48 \%$ admits not having information regarding the impact of environmental and socioeconomic situations. The main conclusion was that ignorance of inadequate solid waste management can bring unfortunate consequences in the economic, social and administrative spheres. Therefore, the support of the authorities and the general population is necessary.
\end{abstract}

Key words: socioeconomic impact; waste management; demographic factor; social factor and environmental education

\footnotetext{
(C) Los autores. Este artículo es publicado por la Revista Campus de la Facultad de Ingeniería y Arquitectura de la Universidad de San Martín de Porres. Este artículo se distribuye en los términos de la Licencia Creative Commons Atribución No-comercial - Compartir-Igual 4.0 Internacional (https://creativecommons.org/licenses/ CC-BY), que permite el uso no comercial, distribución y reproducción en cualquier medio siempre que la obra original sea debidamente citada. Para uso comercial contactar a: revistacampus@usmp.pe.
}

https: 


\section{Introducción}

Tratar de expulsar los sólidos es un problema que abarca ya hace varios ańos a la comunidad, como es en el caso de aquellos residuos sólidos urbanos que vienen a ser el primer nexo de la cadena de problemas y todo empieza cuando el ciudadano solo se preocupa de cómo deshacerse de los residuos sólidos sin el mínimo interés por saber dónde terminarán y las consecuencias futuras que traerá consigo al medio ambiente y no solo es cuestión de los ciudadanos sino de las municipalidades ya que no ejecutan programas alternativos para gestionar dichos residuos sólidos.

Como bien se sabe, cualquier acción humana que integre alteración a la naturaleza ocasiona cierto tipo de residuo. A finales del siglo $\mathrm{XX}$ es cuando la contaminación alcanza niveles elevados. Llega a ser un tema preocupante a nivel mundial y de relevancia para la agenda de los actores políticos y sociales del mundo. Actualmente, ya se perciben las consecuencias de estos problemas ambientales, tales como la crisis energética, el cambio climático, la escasez de recursos, la destrucción de la capa de ozono, así como la contaminación de los suelos, el aire y el agua (MINAM, 2018).

En Colombia, se acumulan aproximadamente 25.999 toneladas diarias de residuos sólidos que anualmente serían 9.488.204 y de las cuales solo 1.880.018 toneladas que se acumulan anualmente son aprovechadas, mejor dicho 6.026 toneladas en 312 días de operación. Las cifras reflejan que solo el $16,54 \%$ de residuos es recuperado (Banco Interamericano de Desarrollo, 2018), causando problemas de contaminación ambiental, incrementando las emisiones de dióxido de carbono y con ello la preocupación de la mayoría de la sociedad por darle un mejor manejo y destino a estos residuos sólidos.

Según los últimos informes de la gestión de residuos del Ministerio del Ambiente, en Lima, se producen más de 8 mil toneladas diarias de residuos sólidos, donde solo el $40 \%$ llega a ser recolectado y el $60 \%$ es botado en sitios informales. Existe insuficiencia con respecto a cómo cubrir los desechos que produce Lima Metropolitana considerando que solo existen cinco rellenos sanitarios. Es por eso, que es indispensable conocer cuál es la gestión que se realiza y cómo esta influye en el medioambiente (MINAM, 2018).

En Lima, se muestra un uso intensivo de vehículos motorizados para desplazarse a los distintos distritos. La cifra refleja que el $73.3 \%$ utiliza el transporte público, el $9 \%$ usa vehículos no motorizados y el $16.6 \%$ utiliza el transporte individual, mostrando consigo una gran necesidad de ejecutar sistemas de gestión para tratar de reducir los impactos ambientales (MINAM, 2018).

En el ámbito local, la ciudad de Moche perteneciente a la provincia de Trujillo, departamento de La Libertad es el tercer departamento con una mayor población en el Perú. Tiene 42 mil habitantes según los datos del INEI, 2018 - 2020 efectuado en enero de 2020. La Municipalidad distrital de Moche tuvo la obligación de elaborar y exhibir la actualización del plan de manejo de aquellos residuos sólidos que el distrito de Moche origina, con el fin de mejorar la gestión, optimizando el rehúso de estos en cada uno de sus etapas. 
Martínez (2017) en su estudio efectuó un análisis de los impactos a nivel social, económico y ambiental de la Gestión de Residuos Sólidos urbanos en unidades cerradas de viviendas en la localidad de Pereira. La metodología empleada en el estudio fue cuantitativa de tipo descriptiva, diseño no experimental. Entre los resultados, el (96,9\%) contestó positivamente que sí podrían reciclar, si es que existieran multas económicas. El $70,9 \%$ de los moradores contestó que los residuos inorgánicos y orgánicos se vienen depositando en los mismos vehículos que hacen la recolección. Así mismo, más de un $25,9 \%$ de los moradores encuestados no sabe qué ocurre con sus residuos sólidos y más de un 2,9\% tiene la creencia de que sus residuos se vienen reciclando.

Luna (2017) en su publicación tuvo por objeto establecer los factores que vienen propiciando y/o inhibiendo las separaciones de residuos en cada hogar. La investigación fue de tipo comparativa. Se obtuvieron los siguientes resultados: la cantidad de basura producida en cada vivienda está vinculada con los lugares donde se hacen las compras, y en donde más basura se genera resulta ser en las superficies más grandes. Una gestión óptima tiene que hacer que el trabajo con los residuos sea más dignificado. Se deben generar puestos de trabajo a los que se incorpore y legalice los empleos no formales.

Peralta et al. (2016) realizaron un estudio que tuvo por objetivo determinar el impacto que un sistema de control de residuos sólidos eficiente en las viviendas puede conseguir en la población, que trasciende más de los cambios físicos de los alrededores. La metodología utilizada en esa investigación fue documental.
Del estudio se obtuvieron los siguientes resultados: más del $94,9 \%$ de las personas encuestadas contestó que posee los servicios de recojo de residuos que realiza el ayuntamiento del municipio.

Así también, más del 47,9\% manifiesta no contar con información; más del $36,9 \%$ no posee información suficiente, y más del 5,9\% revela contar con información suficiente. Respecto a la valoración de la salubridad del espacio donde habitan, más del 4,9\% refiere que es saludable; más del $37,9 \%$ considera regular, y más del 57,9\% declara que no es saludable. Se concluye que la municipalidad presenta grados de contaminantes elevados, resultado de concentraciones de múltiples industrias que evacuan grandes volúmenes de basuras, residuos sólidos, residuos de domicilios e inadecuados manejo a nivel del municipio.

Fernando et ál. (2016) plantearon como objetivo dar los motivos que justifiquen las gestiones de los residuos sólidos, de forma especial el urbano, además estrategias para su desarrollo y las valoraciones de los beneficios que se alcanzan por medio del impacto económico, social y ambiental para la totalidad de regiones del mundo y en especial a los estados en vías de desarrollo. La metodología del estudio fue descriptiva. Se obtuvieron los siguientes resultados: la labor social en este sentido tiene que estar dirigida a la sensibilización. Los hombres deben reducir, reciclar y reutilizar los RSU tanto los que se originan a nivel domiciliario como industrial.

En el aspecto medioambiental se tiene que ahorrar considerablemente energía 
y disminuir las contaminaciones porque no se van a extraer ni procesar recursos naturales vírgenes. Disminuir los gases de efecto invernadero que influyen en los cambios climáticos. Paralizar los agotamientos y explotaciones tanto de los recursos naturales renovables como los no renovables. Referente a los impactos sociales: crear puestos de trabajo y ayuda para que millones de habitantes puedan sostenerse.

Navarro (2016) realizó la evaluación de las situaciones de los manejos de los residuos sólidos en el Cantón Mejía 2015. La metodología de este estudio fue descriptiva correlacional. Se obtuvieron estos resultados: Se pudo recuperar más del 4,48\% de materiales tanto orgánicos como inorgánicos por la sapiencia de los habitantes. En los primeros tres meses de 2016, el porcentaje de recuperaciones aumentó a más del 5,3\% que parece no ser tan elevado, pero en volúmenes de pesos por tonelada va permitir alargamientos de los usos de los rellenos sanitarios.

Privarse del costo asociado a la edificación de un relleno sanitario nuevo, que mínimamente sería $\$ 1^{\prime} 228.172$, suma que contempla la compra de terrenos para la construcción del relleno sanitario, lo cual evitará que más del $4,4 \%$ de residuos inorgánicos y más del 4,27 de residuos orgánicos termine en el relleno ya que son materias que todavía tienen utilidad y significan más de 605 toneladas en cada año.

Del mismo modo, se tomaron estudios nacionales, citando así a Vargas (2017) en su estudio, donde se propuso como objetivo evaluar los elementos económicos sociales que inciden en la Gestión Integral de residuos sólidos en el lugar mencionado. En la investigación se obtuvo los siguientes resultados: respecto al diagnóstico de la gestión integral de residuos sólidos se encontró que el $40 \%$ de la población no conoce la GIRS y el $60 \%$ sí conoce la GIRS. Por otro lado, el $100 \%$ de los pobladores que sí conoce la GIRS ha participado en cursos, talleres, campañas de sensibilización sobre residuos sólidos. Se concluye que en el análisis y evaluaciones hechas por GIRS del lugar indicado, se pudo encontrar que el $60 \%$ de la población conoce la GIRS, mientras que el $40 \%$ desconoce.

Asimismo, Huamaní (2017) en su estudio, en el que se propuso como objeto establecer las propiedades de la GIRS y la propuesta de opciones para que se puedan reaprovechar dichos residuos en la mencionada ciudad; y por último establecer los ingresos y gastos derivados de los reaprovechamientos de los residuos indicados en Juliaca. La metodología fue descriptiva e inferencial. Los resultados que se obtuvieron son: en Juliaca, aproximadamente el $75 \%$ de residuos sólidos proviene de los domicilios, y el $25 \%$, proviene de otros lugares; es decir, de negocios, o de lugares públicos. La gran parte de los pobladores más del $97,9 \%$ no paga ni aporta para la limpieza de la ciudad.

Goncalvez (2017) tuvo por objetivo determinar los impactos socioeconómicos y ambientales con la finalidad de que se formalice las actividades que desempeñan los segregadores de residuos (papeles y plásticos PET) que resultan ser sólidos en dicha ciudad. De la investigación se pudo obtener estos resultados: según los encuestados, el 86\% manifiesta que existen hasta 50 personas que laboran en su sector. Sin embargo, otras personas refieren que se pueden encontrar hasta 
más de 100 personas que se dedican a esta actividad informalmente. De la totalidad de empleados que se lograron identificar, más del 70,9\% $(1,010)$ son segregadores, más del 6,9\% (92) son recolectores, y más del 22,9\% (321) a espacios de almacenes y comercios de dichos residuos.

Según Garriga (2019) los impactos pueden ser definidos como las influencias o efectos dejados por alguien o por algo como consecuencia de todas las acciones o actividades y, obviamente, los impactos sociales pueden ser definidos como las influencias o efectos en la población como efecto de las actividades del hombre al realizar su vida cotidiana.

Por su parte Liberta (2017) señala que los impactos sociales se refieren a los cambios efectuados en la población como resultado de los estudios. Todo resultado final (impacto) es resultado a nivel de propósito o finalidad de los programas que implica una mejora significativa y, a veces, perdurables o sustentables con el paso de los años.

Martínez (2017) sostiene que los comportamientos y el hábito cultural respecto al consumo han establecido de manera importante las cantidades y las calidades de todo residuo sólido, incrementando de esta manera el riesgo para la salud de la población y riesgos medioambientales. Asimismo, se van a generar nuevas alternativas de considerar a todo residuo sólido con posibilidades de ser industrializados. Suele entonces presentarse una problemática como la ausencia de conciencia social y/o conducta sanitaria negativa de parte de los pobladores respeto a la disposición de los residuos porque si son dejados al abandono en vías públicas, parques, riberas de río, playas van a afectar de manera negativa en el paisaje natural y alterar de esta manera los sistemas ambientales y naturales.

Según Echarri en su libro "Ciencias de la Tierra y del Medio Ambiente", un residuo es cualquier tipo de material que esté generado por la actividad humana y que está destinado a ser desechado. La definición de residuo dada por la Organización de las Naciones Unidas para el Desarrollo Industrial en la Guía para la Gestión Integral de los residuos sólidos urbanos es: todo lo que es generado, producto de una actividad y no es de nuestro interés, ya sea por la acción directa del hombre o por la actividad de otros organismos vivos, formándose una masa heterogénea que, en muchos casos, es difícil de reincorporar a los ciclos naturales (Hernández Flechas, 2017).

Por su parte Castro (2017) afirma que es cualquier objeto, material, sustancia o elemento sólido que no tiene ningún valor comercial para el usuario, resultante del consumo o uso de un bien en actividades domésticas, industriales, comerciales, institucionales, de servicios, que el generador abandona, rechaza o entrega y que es susceptible de aprovechamiento o transformación en un nuevo bien, con valor económico o de disposición final.

Los residuos sólidos incluyen todo residuo o desecho en fase sólida o semisólida. También se considera residuos aquellos que siendo líquido o gas se encuentran contenidos en recipientes o depósitos que van a ser desechados, así como los líquidos o gases, que por sus características fisicoquímicas no puedan ser ingresados en los sistemas de tratamiento de emisiones y efluentes y por ello no pueden ser vertidos al ambiente. 
En estos casos, los gases o líquidos deben ser acondicionados de forma segura para su adecuada disposición final. (El Peruano, 2020).

La gestión incontrolada de residuos logra provocar problemas de salud y medioambientales que pueden generar riesgos importantes. Incluso a escala mundial, existen desechos que logran extenderse a grandes distancias, aumentando así la contaminación de la tierra. Además, los residuos sólidos urbanos han adquirido propiedades fisicoquímicas que dificultan su degradación natural. Junto con la escasez de población, la cultura ambiental y los cambios en los hábitos de consumo, la recolección de basura se ha convertido en una tarea importante que requiere un gran gasto de recursos (Couto \& Hernández, 2018).

La recolección y manejo de residuos sólidos se refiere básicamente a un conjunto de acciones claras que se desarrollan desde los aspectos económicos, operativos, administrativos, sociales, de supervisión, monitoreo y educación para que los residuos sólidos municipales puedan ser manejados para el medio ambiente desde su generación hasta su disposición final (Alcocer Quinteros, 2019).

Conceptualmente, la gestión global de los residuos sólidos se compone, fundamentalmente, de un sistema a través del cual se interconectan diferentes procesos. En estos procesos, generalmente, encontramos: separación y almacenamiento; recolección, transferencia y transporte; intermediación y valor agregado; uso de energía (AIDIS, 2018).
Para Bertalanffy (1976), citado por Osorio (2017) un sistema consiste en una red interactiva extremadamente compleja entre una colección de elementos del sistema y las relaciones entre ellos. $\mathrm{Se}$ considera sistema a un sistema compuesto por un conjunto de elementos interrelacionados que dependen de las funciones de otros elementos.

Por su parte Soler (2017) cree que la complejidad de las relaciones que se dan es el motivo de la definición del sistema. Es de estas relaciones que surge el concepto de "sistemas complejos", que se caracteriza por la clara estructura que tienen, algunos de los cuales son parte de ella.

El sistema social tiene como objetivo proporcionar a las personas bienes $y$ servicios para ayudarlos a satisfacer sus necesidades en la vida (Bertoglio, 1993, p.76). Reconocidos por la formulación de la teoría general de los sistemas sociales, estos se basan en la comunicación significativa, y utilizan esta comunicación para componer e interconectar cada uno de sus eventos o acciones. El entorno de estos sistemas es muy complejo porque incluye otros sistemas sociales como la familia, la política, el medio ambiente, la economía, la atención médica, etc. (Segura, 2020).

Por su parte Morasky y Amick (1978), citado por Segura et al. (2020) sostienen que los sistemas sociales suelen ser abiertos y se caracterizan por interactuar con otros sistemas, que proporcionan insumos (entradas) y producen salidas (salidas). Estos sistemas son funciones de otros sistemas fuente, si tienen problemas, se transmiten al sistema principal, y luego el sistema principal puede transmitir estos problemas al sistema receptor para obtener sus resultados o salida. 
El diseño de una política nacional de residuos sólidos debe considerar los siguientes puntos: Diagnosticar el país: se puede comprender el verdadero estado del país en la gestión de residuos sólidos, determinar las ventajas y el atraso del sistema, y su capacidad para realizar cambios importantes que involucren a otros sectores. El diagnóstico debe incluir el valor real de generación de energía, composición física, operación técnica (recolección, transferencia, disposición final), análisis del sistema, análisis legal y regulatorio, plan de compostaje, proyecto de reciclaje, identificación de estados administrativos, económicos y financieros municipales incluyendo humanos, recursos, infraestructura de servicio y soporte y características legales y participación ciudadana en la gestión de residuos sólidos en el país (Ministerio del Ambiente, 2018).

Los desechos o desechos urbanos generados en el ámbito de la gestión municipal, incluida la basura doméstica y los desechos generados por la limpieza y limpieza de lugares públicos, incluidas playas, actividades comerciales y otras actividades urbanas no residenciales pueden ser absorbidos por los servicios públicos. Aseo público, dentro de su jurisdicción (Decreto Legislativo 1278, ley de gestión integral de residuos sólidos, 2016), citado por (AIDIS, 2018). Reciclaje de residuos: El término reciclaje de residuos no aparece en todas las leyes, pero quienes propusieron el término enfatizaron que los residuos constituyen un recurso económico potencial.

Por lo tanto, considerando el reciclaje de sustancias inorgánicas y metales, la generación de energía y la producción de compost, el reciclaje de residuos se dará prioridad. Fertilizantes u otra conversión biológica, recuperación de ingredientes, tratamiento o reciclaje del suelo y otras opciones para evitar la eliminación final. Actualmente, se discute sobre la valorización energética al obtener energía del tratamiento de residuos, pero no se menciona en todas las leyes (AIDIS, 2018).

Manejo de Residuos Urbanos Especiales: Son residuos generados en el ámbito urbano, por su tamaño o características se requiere un manejo especial, tales como residuos de laboratorios de ensayos ambientales y lugares afines, centros de lubricación, centros veterinarios, centros comerciales y gran escala. eventos, tales como no Desechos de conciertos, concentración y movilización de personal temporal, exposiciones, demoliciónoreconstrucción de edificios para proyectos menores dentro de la autoridad del departamento de vivienda y construcción, excepto aquellos dentro de la autoridad. La normativa para la gestión de este tipo de residuos se establece en el reglamento de este decreto legislativo. Los municipios deben fijar tarifas especiales para el manejo y tratamiento de residuos especiales en caso de que sus generadores decidan entregarlos a los servicios públicos de limpieza (El Peruano, 2020).

Gestión integral de residuos: Es toda actividad de gestión técnica destinada a planificar, coordinar, acordar, diseñar, aplicar y evaluar políticas, estrategias, planes y planes de acción para el adecuado manejo de los residuos sólidos (El Peruano, 2020).

El principio de responsabilidad extendida del productor: se alienta a los 
fabricantes, importadores, distribuidores y comercializadores a fabricar o usar productos o empaques que cumplan con los estándares de ecoeficiencia para minimizar la generación de desechos y / o promover el reciclaje de desechos para lograr formas sostenibles de usar los recursos y reducir su impacto sobre el medio ambiente se minimiza. También son responsables de participar en las etapas del ciclo de vida.

Principio de corresponsabilidad: La gestión integral de residuos es una responsabilidad social que requiere la participación conjunta, coordinada $\mathrm{y}$ diferenciada de generadores, operadores de residuos y autoridades municipales.

El principio de protección del medio ambiente y la salud pública: La gestión integral de residuos incluye las medidas necesarias para proteger la salud de las personas y colectivos, en armonía con el pleno ejercicio del derecho básico a vivir en un entorno equilibrado adecuado para la vida y el desarrollo.

Según Palladino (2016) el factor demográfico se debe al crecimiento de la población. Con el tiempo, los cambios demográficos pueden deberse exclusivamente a uno de los siguientes tres factores: fertilidad, mortalidad e inmigración. Estos se denominan "componentes demográficos". Son variables directas que determinan los cambios demográficos, aunque son el resultado de fenómenos más estructurales (sociales, económicos, históricos, culturales, etc.). Estos componentes afectan todos los aspectos de la investigación demográfica: cantidad, estructura y distribución geográfica.
El crecimiento desproporcionado de la población y los cambios en los hábitos de la población, el consumo de productos desechables como orientación y el tratamiento y disposición final inadecuados de los desechos sólidos han traído muchos problemas de contaminación y enfermedades a la población circundante (Vargas, 2015), citado por (Salas López, 2018).

Los factores sociales se definen como actitudes, comportamientos, acciones $\mathrm{y}$ valores que forman comportamientos en el manejo de residuos sólidos. Así mismo, determinar en qué medida estos factores afectan el funcionamiento de las operaciones y procesos de gestión de residuos, y cómo fortalecer los mecanismos en la educación formal (escuelas, instituciones) y la educación no formal (hogares y comunidades) para brindar información suficiente al público. Definir su participación y responsabilidades en los diferentes procesos y operaciones de gestión de residuos sólidos. Dado que la gestión de residuos incluye responsabilidades sociales compartidas, requiere una participación conjunta, coordinada y diferenciada entre generadores, operadores de residuos $y$ municipios. (Ministerio del Ambiente, 2018).

\section{Métodos}

Nos centramos en describir las característicasdelimpactosocioeconómico de la gestión de los residuos sólidos en el distrito de Moche, Trujillo - 2021. El análisis posterior de los mismas también fue transversal o transversal porque los datos se recolectan en un momento dado (Hernández et al., 2014). La tecnología utilizada en este estudio fue la revisión y análisis de documentos, y su herramienta, el registro. 


\section{Resultados y Discusión}

Tabla 1

Determinar las caracteristicas del impacto socioeconómico de la gestión de los residuos sólidos en el distrito de Moche, Trujillo - 2021.

\begin{tabular}{|c|c|c|}
\hline Autor & Fuente & Líneas Identificadas \\
\hline $\begin{array}{l}\text { Martínez, D. } \\
(2017)\end{array}$ & $\begin{array}{l}\text { Revista: "Análisis del } \\
\text { impacto económico, } \\
\text { social y ambiental de la } \\
\text { gestión de residuos sólidos } \\
\text { urbanos en unidades } \\
\text { cerradas de vivienda de } \\
\text { la ciudad de Pereira". }\end{array}$ & $\begin{array}{l}\checkmark \text { Casi el total de las personas respondieron que "SÍ" } \\
\text { reciclarían, si en caso hubiera una sanción económica. } \\
\checkmark \text { Del total de encuestados el } 71 \% \text { menciona que se desecha } \\
\text { en el mismo lugar lo orgánico e inorgánico. } \\
\checkmark \text { El } 26 \% \text { del total de encuestados no tiene conocimiento } \\
\text { sobre los residuos sólidos; mientras tanto el } 3 \% \text { cree que } \\
\text { se recicla. }\end{array}$ \\
\hline Peralta et ál. (2016) & $\begin{array}{l}\text { Revista: “Diagnóstico } \\
\text { socioeconómico y } \\
\text { ambiental del manejo de } \\
\text { residuos sólidos domésticos } \\
\text { en el Municipio de } \\
\text { Haina". }\end{array}$ & $\begin{array}{l}\checkmark \text { Casi el total de encuestados, 95\%, señala que recibe el } \\
\text { servicio del Ayuntamiento de la Municipalidad. } \\
\checkmark \text { En cuanto al conocimiento general sobre el medio } \\
\text { ambiente, el } 48 \% \text { menciona que no tiene información; y } \\
\text { el } 37 \% \text { maneja información insuficiente, y el } 6 \% \text { maneja } \\
\text { información suficiente. } \\
\checkmark \text { En el sentido de la salubridad, una minoría del } 5 \% \text { lo } \\
\text { considera como saludable; } 38 \% \text {, regular, y el } 58 \% \text { lo } \\
\text { señala como no salubre. }\end{array}$ \\
\hline Navarro, N. (2016) & $\begin{array}{l}\text { Revista: "Análisis } \\
\text { so c i o-e co n ó mico } \\
\text { del tratamiento del } \\
\text { tratamiento de residuos } \\
\text { sólidos en el Cantón } \\
\text { Mejía 2015". }\end{array}$ & $\begin{array}{l}\checkmark \text { Se recuperó un aproximado de } 5 \% \text { de materiales orgánicos } \\
\text { e inorgánicos gracias al conocimiento de los ciudadanos, } \\
\text { en el primer trimestre del } 2016 \text {. } \\
\checkmark \text { Aproximadamente, el } 5 \% \text { de residuos inorgánicos como } \\
\text { también el } 4 \% \text { de residuos orgánicos se dirigen al relleno } \\
\text { como basura ya que son materiales que aún poseen utilidad } \\
\text { y significan aproximadamente } 606 \text { toneladas al año. }\end{array}$ \\
\hline
\end{tabular}

De la Tabla 1, se encuentra que, según Martínez (2017) un $24 \%$ de la población encuestada desconoce lo que ocurre con los residuos sólidos generados y solamente un $3 \%$ cree que estos residuos se reciclan. Del mismo modo, según Peralta et ál. (2016) en relación al conocimiento de los pobladores sobre la situación ambiental, el $48 \%$ señala que no dispone de información; el $37 \%$ no dispone de suficiente información, y el $6 \%$ señala que dispone de información apropiada.

Mientras que Navarro (2016) menciona que se recupera un aproximado de 4,5\% de material orgánico e inorgánico por el conocimiento de la población, en el primer trimestre del 2016. Todos estos resultados, a su vez, se sostienen con la teoría de Martínez (2017) quien manifiesta que todo hábito cultural de consumo ha establecido de gran forma las cantidades y las calidades de todo residuo sólido, incrementando, de esta manera, el riesgo de afectar a la salud pública y daños medioambientales.

Asimismo, genera nuevas alternativas para reconocer todo el residuo sólido, un eventual potencial comercial. Se presenta, de esta manera, una problemática en el conocimiento de la población referida a la conciencia pública y/o conducta sanitaria en la disposición de los residuos, no echarlos ni arrojarlos a las vías públicas, en zonas de parque, en las riberas de algún río, playa, mediando de manera perjudicial en la belleza paisajística que nos rodea y de esta manera conseguir 
alterar los sistemas ambientales $y$ naturales.

Por su parte Liberta (2017) indica que los impactos sociales se refieren a los cambios operados en la población como resultado de los estudios realizados. El resultado final (impacto) se da a niveles de propósito o fines de los programas que implica una mejora significativa $y$, a veces, son perdurables o sustentables en los años.

\section{Tabla 2}

Influencia del factor demográfico en el impacto socioeconómico de las gestión de los residuos sólidos en el distrito de Moche, Trujillo - 2021.

\begin{tabular}{|c|c|c|}
\hline Autor & Fuente & Líneas identificadas \\
\hline Fernando et ál. (2016) & $\begin{array}{l}\text { Revista: “Gestión de } \\
\text { los residuos sólidos } \\
y \quad \text { sus impactos } \\
\text { económicos, sociales y } \\
\text { medioambientales". }\end{array}$ & $\begin{array}{l}\checkmark \text { El sentido común debe dirigirse a sensibilizar al } \\
\text { ciudadano en reciclar y reutilizar los RSU sin importar } \\
\text { su procedencia. } \\
\checkmark \text { Al no extraer y procesar los recursos naturales originales } \\
\text { obtenidos de la naturaleza, ayuda a ahorrar mucha } \\
\text { energía y a reducir la contaminación ambiental. } \\
\checkmark \text { Reducir las emisiones de gases de efecto invernadero } \\
\text { que afectan directamente al cambio climático. } \\
\checkmark \text { Detener el consumo y desarrollo de recursos naturales } \\
\text { renovables y no renovables. } \\
\checkmark \text { Ayudar a crear puestos de trabajo y medios de vida para } \\
\text { millones de personas. }\end{array}$ \\
\hline Luna, G. (2017) & $\begin{array}{l}\text { Revista: "Factores } \\
\text { involucrados en el } \\
\text { manejo de la basura } \\
\text { doméstica por parte } \\
\text { del ciudadano". }\end{array}$ & $\begin{array}{l}\checkmark \text { La cantidad de basura generada en cada vivienda está } \\
\text { relacionada con el lugar de compra. } \\
\checkmark \text { La buena gestión debe utilizar la basura para dignificar } \\
\text { el trabajo, crear oportunidades de empleo, incorporar y } \\
\text { legalizar el trabajo informal. }\end{array}$ \\
\hline
\end{tabular}

En la Tabla 2, vemos que Fernando et ál. (2016) mencionan que las labores sociales de acuerdo a lo sostenido deben dirigirse a sensibilizar de la población en la reducción, reciclamiento y reutilización de los RSU, sin importar si se han generado en cada domicilio o en las industrias. Contribuyen a ahorrar considerablemente energía y disminuyen la contaminación del medio ambiente debido a reducir la explotación de utilización de todo recurso natural virgen que es obtenido de la naturaleza.

También se encontró los resultados de Luna (2017) donde indica que una buena gestión, representa la dignificación de la reutilización de la basura, generando empleo, incorporando y legalizando el trabajo informal y que las cantidades de residuos generados en cada vivienda se encuentren relacionados con los lugares en los que se adquieren. Todos estos resultados se sustentan con la teoría expuesta por Palladino (2016) quien explica que el factor demográfico se debe al crecimiento poblacional, el incremento o cambios ocurridos en la población al trascurrir los años es probable que se deba, en exclusividad, a alguno de los siguientes componentes: fecundidad, mortandad y fenómeno migratorio.

A cada uno se le denomina "componente demográfico". Estos componentes son los que ejercen el rol de factores inmediatos y que influyen en el cambio demográfico; no obstante, 
constituyen los resultantes de algún fenómeno más estructural (social, económico, histórico, cultural, etc.). Estos factores van a afectar a cada uno de los fenómenos que son estudiados por la demografía: volúmenes, estructuras y distribuciones geográficas.

Tabla 3

Influencia del factor social en el impacto socioeconómico de la gestión de los residuos sólidos en el distrito de Moche, Trujillo - 2021

\begin{tabular}{|c|c|c|}
\hline Autor & Fuente & Líneas identificadas \\
\hline Vargas, S. (2017) & $\begin{array}{l}\text { Revista: } \quad \text { "Factores } \\
\text { socioeconómicos que } \\
\text { influyen en la inadecuada } \\
\text { gestión integral de residuos } \\
\text { sólidos en el distrito de } \\
\text { Maria". }\end{array}$ & $\begin{array}{l}\checkmark \text { Aproximadamente el } 40 \% \text { del total de ciudadanos } \\
\text { desconoce la Gestión Integral de Residuos Sólidos y } \\
\text { el } 60 \% \text { conoce la GIRS. } \\
\checkmark \text { El total de la población }(100 \%) \text { que conoce la GIRS } \\
\text { ha participado en cursos, talleres, campañas de } \\
\text { sensibilización sobre residuos sólidos. }\end{array}$ \\
\hline Huamaní, C. (2017) & $\begin{array}{l}\text { Revista: } \\
\text { "Análisis socioeconómico } \\
y \quad \text { ambiental del } \\
\text { reaprovechamiento y } \\
\text { disposición final de los } \\
\text { residuos sólidos en la } \\
\text { ciudad de Juliaca, San } \\
\text { Román, Puno-2017". }\end{array}$ & $\begin{array}{l}\checkmark \text { Aproximadamente el } 75 \% \text { de residuos sólidos } \\
\text { proviene de los domicilios, y el } 25 \% \text {, proviene de } \\
\text { otros lugares, es decir de negocios, o de lugares } \\
\text { públicos. } \\
\checkmark \text { Casi el total de la población }(98 \%) \text {, no contribuye } \\
\text { económicamente con la limpieza de la ciudad de } \\
\text { Juliaca, por la cual es una limitante en el tema de } \\
\text { la limpieza. }\end{array}$ \\
\hline Goncalvez. H, (2017) & $\begin{array}{l}\text { Revista: "Efectos } \\
\text { económicos y ambientales } \\
\text { para la formalización de } \\
\text { segregadores de residuos } \\
\text { sólidos de productos } \\
\text { plásticos de categoría } 1 \\
\text { (PET) y papel blanco en } \\
\text { Iquitos, Región Loreto". }\end{array}$ & $\begin{array}{l}\checkmark \quad \text { El } 86 \% \text { manifiesta que existen hasta } 50 \text { personas las } \\
\text { que laboran en el sector de segregadores. } \\
\checkmark \quad \text { De todos los encuestados, el } 71 \% \text { caracterizan a } \\
\text { segregadores, por otra parte, el } 7 \% \text { representan a } \\
\text { recolectores, y el } 23 \% \text { a Locales como almacenes y } \\
\text { del rubro comercial. }\end{array}$ \\
\hline
\end{tabular}

En la Tabla 3, vemos que Huamaní (2017) fue quien sostiene que el $74.9 \%$ de los residuos sólidos que recolecta la municipalidad tiene procedencia de las viviendas, y el $25.0 \%$, cuenta con otra procedencia. Provienen de las empresas, comercios, algún restaurante, hotel, mercado, organizaciones, y del barrido de aceras.

Asimismo, una parte mayoritaria de los pobladores (98\%), no contribuye o no realiza los pagos por el servicio de limpieza en Juliaca, esto sin duda, representa una limitante en la resolución de la problemática de los residuos sólidos. Al respecto Goncalvez (2017) menciona, que el $86 \%$ de los participantes manifiesta que existen hasta 50 personas que laboran en el sector de segregadores.

En tanto, Vargas (2017) refiere que la totalidad de los pobladores conocen la GIRS porque han participado en algún curso, talleres, campañas de sensibilización sobre residuos sólidos que ha conllevado a ser un determinante social. Todo lo expuesto se sostiene con la teoría del Ministerio del Ambiente (2018) quien define al factor social como las actitudes, comportamientos, actos y valores que influyen en la modelación de comportamientos relacionados al tratamiento de los residuos sólidos generados. 


\section{Conclusiones}

Se concluye que el $26 \%$ de todos los encuestados no conoce lo que sucede con dichos residuos y solamente un 3\% cree que estos residuos deben reciclarse. Mientras que en otro estudio, el $48 \%$ reconoce no tener información suficiente para contribuir con gestionar estos residuos sólidos.

Respecto a las labores sociales, se concluye que deben estar direccionadas a lograr sensibilizar a la población para disminuir, reciclar y reutilizar los
Residuos Sólidos Urbanos que se generan en los domicilios y en las industrias.

Asimismo, se ayuda a ahorrar de manera importante el consumo de energía y disminuir los niveles de contaminación ambiental ya que se va a extraer y procesar cada recurso natural virgen que se encuentra en naturaleza.

Finalmente, se concluye que, el 74.9 $\%$ de los residuos sólidos recogidos por el municipio se origina en los domicilios, y el $25.0 \%$, tiene otra procedencia. Proviene de la industria, comercio, restaurantes, hoteles, mercados, y organizaciones.

\section{Agradecimiento}

A la Universidad César Vallejo por la oportunidad de realizar estas investigaciones conjuntas.

\section{Referencias}

Acosta, A., Atiencia, M., \& Guanopatin, A. (2017). Teorias de la Investigación. Universidad Central del Ecuador.

AIDIS. (2018). Gestion Integral de Residuos Solidos Urbanos. Diseńo, formación y revisión. Proper Mx.

Alcocer Q, P. R. (2019). Mejoramiento de la gestión integral de los residuos sólidos urbanos en el cantón de Quevedo, Euador. Revista Universidad y Sociedad, 11(5), 362-367.

Banco Interamericano de Desarrollo. (2018). Situación de la gestión de residuos sólidos en América Latina y el Caribe. Normal.

Castro, A. (2017). Qué es un residuo Sólido. Bazero.
Couto, I., \& Hernández, A. (2018). Participación y rendimiento de la iniciativa privada en la gestión integral de los residuos sólidos urbanos en la frontera México-Estados Unidos. Gestión y Politica Pública.

El Peruano. (2020). Decreto Legislativo No 1278. Diario Oficial del Bicentenario.

Fernando do Rosario, J. B., Concepción Toledo, D. N., Barrios Castillo, G., \& Gonzáles Suárez, E. (2016). Gestión de los residuos sólidos y sus impactos económicos, sociales y medioambientales. Revista Centro Azúcar.

Garriga , E. (2019). Impacto Social: Un modelo en base a capacidades. Política de Empresa de EADA. 
Goncalvez, H. P. (2017). Efectos económicos y ambientales para la formalizaciòn de segregadores de residuos sólidos de productos plásticos de categorìa 1 (PET) y papel blanco en Iquitos, Región Loreto. Scielo.

Hernández, S. (2017). Reflexiones sobre la importancia económica y ambiental del manejo de residuos en el siglo XXI. Dialnet.

Huamaní , C. (2017). Análisis socioeconómico y ambiental del reaprovechamiento y disposición final de los residuos sólidos en la ciudad de Juliaca, San Román, Puno - 2017. Scielo.

Liberta , B. E. (2017). Impacto, impacto social y evaluación del impacto . Acimed.

Luna, G. (2017). Factores involucrados en el manejo de la basura doméstica por parte del ciudadano. Departamento de Psicologia Social.

Martínez , D. (2017). Análisis del impacto económico, social y ambiental de la gestión de residuos sólidos urbanos en unidades cerradas de vivienda de la ciudad de Pereira. Scielo.

MINAM. (2018). Gestión de Residuos Sólidos. Lima, Perú: Ministerio del Ambiente. . https://www.gob.pe/ minam

MINAM. (2018). Perú limpio Proyectos Rellenos Sanitarios: Avances. Lima. https://www.gob.pe/minam
Ministerio del Ambiente. (2018). Residuos sólidos. Ministerio del Ambiente.

Navarro, G. M. (2016). Análisis socio-económico del tratamiento del tratamiento de residuos sólidos en el Canton Mejía 2015. Scielo.

Osorio, F. (2017). Introducción a los Conceptos Básicos de la Teoría General de Sistemas. Cinta de Moebio.

Palladino, A. (2016). Introducción a la Demografía. Estudios $S C$.

Peralta, E., Del Rosario , A., \& Vélez, C. (2016). Diagnóstico socioeconómico y ambiental del manejo de residuos sólidos domésticos en el Municipio de Haina. Ciencia y sociedad.

Salas, R. (2018). Factores que influyen en el manejo de los residuos sólidos municipales, Pomacochas, Amazonas. Rev. de investig. agroproducción sustentable.

Segura, A. (2020). Referentes mundiales en sistemas de gestión de residuos sólidos. Espacios.

Soler, Y. (2017). Teorías sobre los sistemas complejos. Revista A\&D.

Vargas, S., \& Oliva, M. (2017). Factores socioeconómicos que influyen en la inadecuada gestión integral de residuos sólidos en el distrito de María. UNTRM-A. 